\title{
GRUPOS REALIZADOS POR ENFERMEIROS NA ÁREA DE SAÚDE MENTAL
}

\author{
Groups carried out by professional nurses in the mental health field \\ Grupos realizados por enfermeros en el área de salud mental
}

Luciene Simões Spadini

Maria Conceição Bernardo de Mello e Souza

\begin{abstract}
Resumo
Este artigo teve por objetivo realizar uma revisão da literatura nacional sobre 0 trabalho em grupos realizados por enfermeiros na área de saúde mental, no período de janeiro 1994 a abril de 2004. A identificação das fontes foi realizada através dos sistemas informatizados de busca Literatura Latino-Americana de Ciências da Saúde (LILACS) e o Banco de Dados Bibliográficos da USP - Catálogo On-line Global (DEDALUS). Foram encontrados 38 trabalhos, 17 da área de enfermagem e seis especificamente sobre o trabalho em grupo realizado por enfermeiros na área de saúde mental. Os seis artigos foram analisados segundo: 0 tipo de grupo; a coordenação; os integrantes; local e objetivo da formação. Constatou-se que há a necessidade do preparo do enfermeiro para a realização dessa atividade, diante da relevância dessa estratégia para a assistência de enfermagem em saúde mental. Há a necessidade de novas publicaçōes mediante ao número pequeno de publicações sobre a temática.
\end{abstract}

Palavras-chave: Enfermagem. Saúde Mental. Enfermagem Psiquiátrica.

\section{Abstract}

This article had the purpose of to carry out a national literature revision about work in groups carried out by nurses in the mental health field, in the period between January of 1994 and April of 2004. The identification of the sources was carried out through the computerized systems of search Latin American Literature of Health Science (LILACS) and the Bibliographical Data Base from USP - Global Online Catalogue (DEDALUS). It had been found 38 paper works, 17 in the nursing field and six specifically about the work in group carried out by nurses in the mental health field. The six articles had been analyzed according to: type of group; coordination; the integrants; place and objective of the formation. It was evidenced that it has the necessity of the preparation of the nurse for the accomplishment of this activity, face to the relevance of this strategy for the assistance of nur sing in mental health. It has the necessity of new publications about this thematic because of the small number that are available nowadays.

\section{Resumen}

Este artículo tuvo por objetivo realizar una revisión de la literatura nacional sobre el trabajo en grupos realizados por enfermeros en el área de salud mental, en el período de enero de 1994 a abril de 2004. La identificación de las fuentes fue realizada a través de los sistemas informatizados de búsqueta Literatura Latinoamericana de Ciencias de la Salud (LILACS) y el Banco de Datos Bibliográficos de la USP - Catálogo on-line Global (DEDALUS). Fueron encontrados 38 trabajos, 17 del área de enfermería y seis específicamente sobre el trabajo en grupo realizado por enfermeros en el área de salud mental. Los seis artículos fueron analizados según: el tipo de grupo; la coordinación; los integrantes; local y objetivo de la formación. Se constató que existe la necesidad de la preparación del enfermero para la realización de esa actividad, delante de la relevancia de esa estrategia para la asistencia de enfermería en salud mental. Existe la necesidad de nuevas publicaciones mediante al número pequeño de publicaciones sobre la temática.
Keywords:

Nursing. Mental Health. Psychiatric Nursing.
Palabras clave:

Enfermería. Salud Mental. Enfermería Psiquiátrica. 


\section{INTRODUÇÃO}

0 ser humano não vive só. Para se desenvolver e sobreviver necessita dos grupos, nos quais podem ser trabalhadas as relações e existe a possibilidade de se obter ajuda e troca de experiências necessárias para a vida. Os grupos proporcionam, entre outras coisas, oportunidades para o enfrentamento dos medos, das angústias e culpas e dos conflitos presentes no cotidiano do homem.

0 homem está capacitado a funcionar de forma mais eficiente em grupos do que sozinho. Desse modo, há uma tendência ao autoconhecimento nos grupos e, particularmente, neles é que se pode observar como as necessidades humanas levam os homens a se articularem grupalmente (1). Um estudo com grupos de portadores de esquizofrenia, que fazem uso de clozapina, e seus familiares mostrou nos resultados encontrados que houve um desempenho positivo do trabalho grupal no cotidiano de pacientes e familiares, evidenciando transformações importantes na qualidade de vida dos mesmos ${ }^{(2)}$.

Portanto, existem grupos de todos os tipos. Existe uma primeira subdivisão que se faz necessária em grupos primários e secundários. A família seria um exemplo de grupo primário e a escola, o trabalho e as instituições, de grupos secundários. Nos grupos, cada ser encontra um lugar, um papel, que por sua vez constitui nossa maneira de ser. Neste espaço desempenhamos nosso papel, segundo a nossa história (3).

Os seres humanos têm seu destino ligado ao funcionamento de grupos e não se pode ter uma visão do homem, sem se ter uma visão lúcida dos grupos humanos com os quais eles interagem ${ }^{(4)}$. Um conjunto de pessoas constitui um grupo, um conjunto de grupos constitui uma comunidade e um conjunto de comunidades que interagem, representa uma sociedade. Como o indivíduo passa a maior parte de sua vida convivendo e interagindo em grupos, tornase importante o conhecimento e a utilização da estratégia grupal (5).

Grupo é o resultado da dialética entre a história do grupo e a história dos integrantes do mesmo, com seus mundos internos, suas projeções e transferências no caminhar da história da sociedade em que estão inseridos ${ }^{(3)}$. A definição de grupo é vaga e imprecisa, pois pode ser o conjunto de duas ou três pessoas, como também uma família, uma gangue, uma classe ou um grupo terapêutico ${ }^{(6)}$. Um grupo não é um somatório de pessoas, mas uma entidade com mecanismos específicos, próprios e com leis, no qual todos os integrantes estão unidos para 0 alcance de um objetivo comum ${ }^{(5)}$.

Conforme Zimerman ${ }^{(6)}$, é relevante apontar alguns estudiosos do movimento grupalista, como Pratt que, em 1905, criou o método de classes coletivas, o qual subsidiou posteriormente a organização dos Alcoólicos Anônimos. Em 1921, Freud traz como contribuição às noções sobre a psicologia das multidões, os grandes grupos artificiais como igreja e exército, os processos identificatórios que vinculam pessoas e os grupos, as lideranças que influem na coesão e desagregação dos grupos ${ }^{(6)}$.

Em 1930, Moreno introduziu a importante técnica do psicodrama. Já Lewin criou a expressão dinâmica de grupo. Em 1948, Foulkes inaugurou a prática da psicoterapia psicanalítica de grupo, sendo considerado líder mundial da mesma. Pichon Rivière tornou-se 0 grande nome na área dos grupos operativos, com contribuições originais, mundialmente aceitas e praticadas por vários profissionais. Na década de 40 , Bion criou e difundiu conceitos totalmente originais acerca da dinâmica de grupo ${ }^{(6)}$.

A Escola Francesa, na década de 60 , foi um movimento surgido no período em que as grupoterapias começam a adquirir referenciais específicos e identidade própria. A Escola Argentina foi o movimento no qual surgiu a denominada concepção "psicanálise compartida" e "psicanálise das configurações vinculares". Considerando toda esta evolução relativa ao grupo, o Brasil atualmente está em busca de novos caminhos de uma assistência mais ampla com recursos baseados na dinâmica grupal ${ }^{(6)}$.

A principal diferença entre os grupos ocorre na sua finalidade, isto é, para que eles foram criados e compostos. Em relação aos tipos de grupos, eles são classificados em operativos e psicoterápicos. Os grupos operativos abrangem os demais tipos de grupos, como os terapêuticos e envolve os campos: de ensinoaprendizagem, institucionais, comunitários e terapêuticos. Os grupos psicoterápicos / terapêuticos são os que se destinam à aquisição de insight, dos aspectos inconscientes dos indivíduos e da totalidade grupal, como os grupos psicoterápicos propriamente ditos e os de auto - ajuda ${ }^{(6)}$.

Os motivos para utilização da estratégia de grupos existem no sentido de mobilizar, estimular, educar, treinar para o trabalho e para a vida em sociedade, conscientizar, assim como, abordar problemas de relacionamento. Seu uso deve-se em parte, às vantagens econômicas, em tratar várias pessoas ao mesmo tempo e também, pela economia de recursos 
humanos. Os grupos têm a capacidade de recriar ambientes familiares, sociais, possibilitando 0 desenvolvimento de habilidades, de criações e desse modo, ele é um instrumento terapêutico eficiente ${ }^{(7)}$.

0 enfermeiro desenvolve todo o seu trabalho em grupo citando-se os seguintes exemplos: durante a passagem de plantão, na execução de trabalhos educativos, nos grupos de discussão de casos, ou em disciplinas, como parte da aprendizagem. A partir da década de 80 surgiram publicações sobre atividades grupais desenvolvidas por enfermeiras. Porém, os trabalhos não se referem ao preparo do enfermeiro para tal atividade, ao passo que isso é fundamental para o mesmo trabalhar com essa abordagem, principalmente quanto às suas variáveis, técnicas de condução e dinâmica interna ${ }^{(8)}$.

Em instituição ou serviço onde existe a proposta do trabalho em equipe multidisciplinar, torna-se claro a função do enfermeiro no trabalho em grupo, pelo conhecimento que ele tem sobre aspectos teóricos e práticos do trabalho grupal. A atividade grupal tem sua efetividade comprovada, mas ainda é bastante limitada. Talvez, uma possível explicação possa ser a carência de profissionais capacitados para esse tipo de intervenção (2).

0 processo de implementação dos novos instrumentos de assistência aos doentes mentais ainda é recente, tornando-se necessário novos estudos que redirecionem as novas formas de intervenção, como a estratégia grupal, no tratamento desses pacientes, assim como, 0 preparo do profissional para poder utilizar esse recurso que, sendo implantado nas comunidades como um todo, ajudaria a limitar 0 número de reinternações, pois atenderia de modo mais amplo à essa demanda.

Se o paciente recebe um bom atendimento em grupo por um profissional qualificado como suporte, certamente ele poderá compreender vários aspectos de sua doença e tratamento, receberá o apoio emocional necessário e isso fará com que tenha condições de se manter bem e não ser internado, uma vez que o objetivo maior da assistência é mantê-lo fora do hospital.

Um estudo aponta pelo relato de familiares, que seus entes doentes antes do grupo tinham a vida estacionada, várias dificuldades eram mais destacadas como nas áreas de relacionamento, cognição e atividades sociais. Foi constatada neste estudo a participação dos doentes no grupo melhorou significativamente os sintomas negativos da doença (2).

Em seu processo de trabalho, a Enfermagem precisa antes de tudo, reconhecer a importância do trabalho grupal, porque ainda é uma área de atuação pouco explorada pelos enfermeiros, não somente na saúde mental, mas em todas as outras áreas. Assim, ele poderá buscar o conhecimento necessário para a aplicação dessa estratégia, objetivando-se a melhor assistência aos pacientes e seus familiares, e entendendo que 0 atendimento por grupos é. hoje, uma das principais vertentes no tratamento dos portadores de transtorno mental ${ }^{(8)}$. Desse modo, a proposta deste trabalho é fazer um estudo bibliográfico a respeito do tema, no sentido de identificar os tipos de grupos realizados por enfermeiros na área de saúde mental.

\section{OBJ ETIVO}

Realizar uma revisão da literatura nas publicações em periódicos nacionais sobre o trabalho em grupos realizados por enfermeiros na área de saúde mental, no período de janeiro de 1994 a abril de 2004.

\section{METODOLOGIA}

Este estudo teve por finalidade realizar uma revisão das publicações em periódicos nacionais sobre 0 trabalho em grupo realizado por enfermeiros na área de saúde mental,no período de janeiro de 1994 a abril de 2004. A revisão da literatura tem por objetivo conhecer as publicações sobre um determinado assunto em um determinado período. E, ela se define pela leitura, relacionamento, fichamento e arquivamento de tópicos importantes para a pesquisa em questão. Uma revisão constituí-se pelas seguintes fases: identificação, localização, obtenção, fichamento, arquivamento e redação do trabalho ${ }^{(9)}$.

As fontes bibliográficas foram identificadas através dos sistemas informatizados de busca denominados Literatura Americana de Ciências da Saúde (LILACS) e Banco de Dados Bibliográficos da USP (DEDALUS). Para isso, foram utilizadas as seguintes palavras chaves: Enfermagem, Grupos, Saúde Mental e Psiquiatria. Posteriormente, os trabalhos foram selecionados, lidos, fichados e analisados conforme metodologia da pesquisa bibliográfica, com vistas a conhecer as contribuições dos trabalhos sobre o tema. Os dados para este estudo foram coletados no mês de abril de 2004.

De acordo com as palavras chaves utilizadas, foram encontrados 38 trabalhos sobre a temática grupos. Desses estudos, quinze eram da área da Terapia Ocupacional, Medicina e Psicologia em Saúde Mental. Dezessete eram da área de Enfermagem abordando 
outras questões generalizadas sobre 0 trabalho em grupo. E seis trabalhos eram específicos do trabalho em grupos realizados por enfermeiros na área de saúde mental.

De acordo com o objetivo do presente estudo, foram selecionados apenas os seis trabalhos relacionados com a temática grupos em enfermagem na área de saúde mental. Eles foram analisados segundo: (a) a modalidade, isto é, o tipo de grupo; (b) seu coordenador, ou seja, qual profissional coordenava o grupo; (c) seus integrantes (pacientes, familiares, alunos, outros); (d) local em que os mesmos foram realizados; e (e) qual era 0 objetivo da formação do grupo. A discussão dos dados foi realizada com embasamento na literatura sobre o trabalho em grupos.

\section{RESULTADOSE DISCUSSÃO}

A intenção neste estudo é apresentar os dados coletados e fazer um discussão sobre o tema trabalho em grupos realizados por enfermeiros na área de saúde mental. Os artigos encontrados referem-se, como já mencionado ao período de janeiro de 1994 a abril de 2004. 0 Quadro 1, apresentado a seguir, mostra os dados encontrados nas publicações estudadas.

Conforme podemos visualizar neste Quadro, encontramos seis publicações sobre o tema investigado, no período de 10 anos. Neste total, estão incluídos os trabalhos publicados na íntegra e resumos. Dentre as seis publicações selecionadas, em relação a modalidade de grupo, um deles trata de grupo de suporte / apoio, três outros referem-se a grupos operativos, uma publicação relaciona-se a grupo de auto - ajuda e a última delas, relata experiência de grupo realizado em sala de espera.

0 grupo de suporte / apoio pode ajudar pessoas em períodos de adaptação à mudanças, à novas situações ou na manutenção delas, assim como no tratamento de crises. Esta modalidade de grupo parece ser de utilidade devido aos benefícios que traz aos seus participantes ${ }^{(8)}$. 0 principal objetivo dos grupos de apoio é ajudar seus membros a enfrentar 0 estresse da vida e o foco deste tipo de grupo está sobre os pensamentos, sentimentos e comportamentos disfuncionais, para os quais são oferecidos apoio emocional e informações críticas para o aumento das capacidades de seus membros para enfrentamento e solução dos problemas, reforçando o sistema de apoio entre os pacientes ${ }^{(10)}$.

Quanto à modalidade grupo operativo encontrado em dois artigos, seu objetivo pode estar centrado em uma tarefa que pode ser 0 aprendizado, as dificuldades, a cura, o diagnóstico e outras ${ }^{(11)}$. A conceituação de grupo operativo é abrangente e a sua aplicação extensa. Tanto que, muitos consideram-no como um contingente dos demais grupos, inclusive os grupos terapêuticos e mesmo, os grupos psicanalíticos. Os grupos operativos cobrem os campos do ensinoaprendizagem, institucionais, comunitários e terapêuticos ${ }^{(5)}$. Em conformidade com esta posição, os grupos operativos encontrados no presente estudo foram dois no campo terapêutico e um no campo do ensino - aprendizagem ${ }^{(6)}$. No campo do ensino aprendizagem, a utilização do grupo operativo como técnica para trabalhar com pequenos grupos de estudantes têm sido utilizado no sentido de que 0 referencial Pichoniano, vem abrindo a possibilidade desse constante repensar e, fundamentalmente, de refazer a prática do ensinar, incorporando estudantes e professores como sujeitos críticos e criativos que possam transformar 0 cotidiano ${ }^{(12)}$.

Em relação aos grupos de auto - ajuda, descrito em um artigo, eles são uma modalidade do grupo operativo terapêutico e consistem no fato de serem

Q uadro 1:

Distribuição dos artigos em relação a modalidade, coordenador, integrantes, local em que é realizado e objetivo do grupo. Ribeirão Preto, 2004.

\begin{tabular}{|c|c|c|c|c|c|}
\hline Artigo & Modalidade & Coordenador & Integrantes & Local & Objetivo do grupo \\
\hline 1 & Suporte/Apoio & Enfermeiro & Paciente cirúrgico & Clínica cirúgica & Aliviar tensão, ansiedade e medo durante a internação \\
\hline 2 & Operativo & Enfermeiro & Paciente psiquiátrico & Hospital & Enfrentamento do contexto social \\
\hline 3 & Operativo & Enfermeiro & Mulheres depressivas & Não informou & Expressão vivencial das mesmas \\
\hline 4 & Espera & Enfermeiro & $\begin{array}{c}\text { Familiares de pacientes } \\
\text { psiquiátrico }\end{array}$ & $\begin{array}{l}\text { Ambulatório de } \\
\text { saúde mental }\end{array}$ & Discutir pontos importantes do contexto familiar \\
\hline 5 & Auto - ajuda & Facilitadora & $\begin{array}{l}\text { Paciente psiquiátrico, } \\
\text { seus familiares e amigos }\end{array}$ & $\begin{array}{c}\text { Imóvel comum } \\
\text { para a realização } \\
\text { dos encontros }\end{array}$ & $\begin{array}{l}\text { Desenvolvimento de atitudes mais ativas nos } \\
\text { relacionamentos e diversos segmentos da sociedade }\end{array}$ \\
\hline 6 & Operativo & Enfermeiro & Alunos & $\begin{array}{l}\text { Escola de } \\
\text { Enfermagem }\end{array}$ & Ensino \\
\hline
\end{tabular}


grupos de formação espontânea, de pessoas que se sentem identificadas por características semelhantes entre si. A utilização terapêutica do grupo de auto - ajuda merece destaque, tanto pela sua eficácia, como pelo largo âmbito de sua aplicação e expansão ${ }^{(5)}$. Os grupos de auto - ajuda são organizados ao redor de uma experiência comum, podendo ou não receber consultoria de um provedor da saúde, como um enfermeiro. Porém, eles são operados pelos seus membros ${ }^{(10)}$.

Quanto ao grupo realizado em sala de espera, descrito em uma das publicações, geralmente ele é criado para preencher o tempo ocioso das pessoas que esperam por atendimento ou pelo familiar que está sendo assistido nos serviços de saúde. Nas instituições que oferecem atendimento psicológico gratuito, vem ocorrendo uma procura cada vez maior pelo serviço. Assim, fica evidente a necessidade de diversificação e busca de novas soluções para esta clientela. A publicação menciona que, a partir desse quadro foi feita a opção por criar um grupo de espera para a psicoterapia realizada na instituição. Para alguns pacientes esta breve experiência foi suficiente para suas necessidades e se constatou que o grupo em sala de espera possibilita uma maior disponibilidade para 0 trabalho grupal com maior confiança em sua eficácia e benefícios. A publicação ainda destaca que grupo de espera é um instrumento capaz de cumprir os objetivos de sua idealização e possibilita novas formas de pensar 0 trabalho institucional ${ }^{(13)}$.

$\mathrm{Na}$ análise dos seis trabalhos selecionados com relação à coordenação, verificou-se que a enfermeira aparece como coordenadora em cinco desses estudos. 0 recurso grupal tem sido reconhecido pelo enfermeiro pelo seu valor terapêutico e fundamental para melhora na qualidade da assistência.

Na saúde mental as atividades desenvolvidas em grupos são muito utilizadas e constituem-se em estratégias eficazes para a Enfermagem, na assistência ao paciente e seus familiares. Sendo assim, a enfermeira deve buscar 0 embasamento necessário para realizar essas atividades de forma competente e efetiva. É importante que 0 enfermeiro busque 0 conhecimento necessário para desenvolver a função de coordenador de grupo. Mas também, é preciso dar importância a percepção de si mesmo e ao modo de relacionamento mantido com o grupo.

0 enfermeiro que conta apenas com sua experiência poderá ter dificuldades para coordenar grupos. Portanto, ele estará mais seguro se tiver conhecimentos sobre a dinâmica humana e dos grupos
(14). Quando exerce a função de coordenador de grupos e o faz com competência, 0 enfermeiro contribui para que a Enfermagem ganhe valor e reconhecimento, aspectos que são importantes na conquista de seu espaço enquanto profissão.

Em um dos artigos examinados, a enfermeira exerce o papel de facilitadora do movimento grupal. Muitos grupos de auto - ajuda formam-se a partir do estímulo integrador de um profissional que inicialmente coordena 0 grupo, até o momento em que o mesmo possa caminhar sozinho, quando o profissional afastase, mantendo-se disponível para o grupo que ele ajudou a formar. Assim, o profissional pode ser considerado como facilitador do grupo ${ }^{(5)}$.

Em relação aos integrantes dos grupos na análise das publicações deste estudo, eles variam desde pacientes psiquiátricos até seus familiares e amigos. Em uma dessas publicações, o grupo está destinado aos pacientes em situação de estresse em uma clínica cirúrgica. Em outra publicação, o grupo é direcionado para alunos de graduação em enfermagem.

Verifica-se nos artigos que, a maioria dos grupos na área de psiquiatria são realizados com os pacientes. Assim, questiona-se os limites da prática com relação ao atendimento do familiar num contexto em que a participação da família é fundamental para a evolução do tratamento do doente. Por isto, deveria haver maior utilização da estratégia grupal para 0 atendimento dessa clientela (família), no sentido de dar suporte a mesma e a ajudar também, no manejo com seus parentes doentes. É necessário considerar as experiências singulares desses familiares na convivência com o paciente de transtorno mental, como estratégia para a integração e re-imersão social do portador de transtorno mental na sociedade ${ }^{(15)}$.

Desse modo, torna-se necessário ampliar as estratégias que visem dar atendimento às famílias de modo que recebam 0 apoio adequado e possam contribuir melhor para o processo de tratamento de seu parente doente. Neste sentido, a realização de grupos pode ser um ótimo recurso para alcançar esse propósito.

Um outro aspecto a ser destacado relaciona-se ao ensino-aprendizagem em grupo, realizado com os alunos de graduação em enfermagem em disciplina de dinâmica de grupo, uma abordagem encontrada em um dos artigos. Faz-se necessário ressaltar a importância dessa disciplina na formação do enfermeiro, pois ele desenvolve todo 0 seu trabalho em grupos, no âmbito da equipe de enfermagem, em trabalhos educativos e mesmo, na coordenação de grupos na assistência de enfermagem ${ }^{(8)}$. 
Quanto aos locais de realização dos grupos nas publicações consideradas, um é em hospital geral (clínica cirúrgica), um ocorreu em local do hospital não especificado, um deles não especificou o local, um foi realizado em ambulatório de saúde mental, um em uma universidade e outro em um imóvel específico para as realizações das reuniões. Destes dados depreende-se que a realização dos grupos ocorre de um modo geral em instituições de saúde hospitalar e ambulatorial, exceto dois que são realizados em outros locais.

Pode-se inferir que a psiquiatria atual define a comunidade como espaço social de intervenção mais amplo, devido aos recursos institucionais, humanos, culturais, religiosos, famílias, amigos, educacionais que, somados aos esforços de prevenção da psiquiatria, podem curar e reabilitar os portadores de transtorno mentais ${ }^{(16)}$. Portanto, corrobora-se a importância da utilização do recurso grupal na comunidade, uma vez que esta atividade pode contribuir para que o paciente fique na comunidade, na medida em que compreendendo melhor sua condição, melhora sua adesão ao tratamento e portanto, o número de internações provavelmente diminuirá.

Ao observar-se os objetivos dos grupos, isto é a finalidade de sua formação, verifica-se que 0 artigo 1 aponta como finalidade, 0 alívio de tensão, ansiedade e medo e o tipo de grupo utilizado é de suporte / apoio. Nos artigos 2 e 4, o objetivo está ligado ao enfrentamento e a discussão do contexto social e familiar, sendo utilizadas as modalidades de grupos operativo e de espera, respectivamente. Como já foi mencionado neste estudo, o grupo operativo têm uma vasta aplicação podendo encaixar-se para tal objetivo. E o grupo em sala de espera é vantajoso para qualquer finalidade, uma vez que se ocupa um tempo, que seria desperdiçado, no desenvolvimento de uma atividade produtiva.

No artigo 3, o objetivo é a livre expressão vivencial de mulheres deprimidas utilizando-se também, 0 grupo operativo como espaço para esta finalidade. Em um site, a definição da técnica de grupo operativo de Enrique Pichon Rivieri é apontada como a integração de aspectos vivencias e intelectuais em que os participantes do grupo, ao mesmo tempo discutem e estudam os diversos obstáculos que surgem em si e nos demais sendo portanto, uma tipo de grupo muito aplicável para este objetivo e a clientela referida no artigo ${ }^{(17)}$.

Em relação ao artigo 5, o objetivo do grupo é desenvolver entre os seus membros atitudes mais ativas no relacionamento com as pessoas e também, nos diversos segmentos da sociedade utilizando-se do grupo de auto - ajuda. Nestes grupos, os participantes trocam experiências e informações úteis para 0 enfrentamento de seus problemas e dificuldades. Assim, diante do exposto, há concordância com 0 objetivo do grupo referido no artigo, pois há uma relação de ajuda mútua entre os membros para melhorarem sua situação de vida (18).

Quanto ao artigo 6, o objetivo do grupo é o ensinoaprendizagem sobre a dinâmica de grupos utilizandose 0 grupo operativo como estratégia. 0 grupo operativo cobre o campo do ensino-aprendizagem e é muito utilizado com esta finalidade. A dinâmica de grupo é um campo de estudo, de trabalho e de pesquisa integrado às ciências sociais e um instrumento de aperfeiçoamento do funcionamento das pessoas, dos grupos e das sociedades humanas. Seu objetivo é buscar o trabalho cooperativo e em equipe ${ }^{(4)}$.

\section{CONSIDERAÇÕES FINAIS}

0 recurso grupal é uma estratégia importante nas ações de enfermagem, no sentido de favorecer a melhoria na qualidade da assistência ao paciente e seus familiares. Assim, de acordo com os resultados do presente estudo pôde-se identificar que, as modalidades de grupo mais utilizadas por enfermeiros na área da saúde mental são os grupos operativos e de suporte / apoio e os grupos em sala de espera. 0 enfermeiro é coordenador na maioria deles e aparece também como facilitador do grupo de auto - ajuda. Destaca-se que, o grupo operativo foi o tipo de grupo mais utilizado pelos enfermeiros.

Os grupos são realizados em sua maioria em instituições de saúde e um deles em uma instituição de ensino superior. Destaca-se a necessidade do aumento da sua realização na comunidade, como forma de atendimento aos princípios da atual Política de Saúde Mental. Os grupos são direcionados à pacientes em sua maioria, mas também aos familiares e amigos dos portadores de transtornos mentais. 0 grupo também pode ser adotado para outros tipos de clientela ressaltando-se dentre elas, os alunos de graduação em enfermagem.

Em sua maioria, os objetivos dos grupos visam trabalhar com os participantes as situações emocionais vivenciadas pelos mesmos, bem como o seu enfrentamento. Entretanto, o objetivo de um dos grupos volta-se para o ensino-aprendizagem em um curso de graduação em enfermagem.

As autoras das publicações pesquisadas de uma forma geral, consideram a importância do preparo do 
enfermeiro para a realização das atividades grupais e também, para as supervisões periódicas. Mencionam ainda, a relevância do trabalho grupal para a assistência de enfermagem em saúde mental e 0 benefício que este recurso traz ao tratamento de pacientes e seus familiares.

Com este estudo foi possível verificar que há poucas pesquisas publicadas sobre a temática. Assim, podese concluir que novos estudos sobre o tema precisam ser realizados e até mesmo, é necessário que se aumente na prática, a utilização deste recurso por enfermeiros da área psiquiátrica e saúde mental, para a melhoria da qualidade da assistência prestada.

\section{Referências}

1. Osório LC, organizador. Grupoterapia hoje. Porto Alegre (RS): Artes Médicas; 1989.

2. Durão MAS. Grupo de acompanhamento de pacientes portadores de esquizofrenia medicados com clozapina e de seus familiares: 0 impacto sobre o cotidiano de suas vidas [ dissertação de mestrado]. Ribeirão Preto (SP): Escola de Enfermagem de Ribeirão Preto/USP; 2004.

3. Freire M. 0 que é um grupo? In: Grossi EP, Brodim J, organizadoras. A paixão de aprender. 8ª ed. Rio de Janeiro (RJ): Vozes; 1996.

4. Busnello ED. Dinâmica de grupo: fundamentos, delimitação do conceito, origens e objetivos. In: Osório LC, organizador. Grupoterapia hoje. Por to Alegre (RS): Artes Médicas; 1986.

5. Zimerman DE. Fundamentos teóricos. In: Zimerman DE, Osório LC, organizadores. Como trabalhamos com grupos. Porto Alegre (RS): Artes Médicas; 1997.

6. . Fundamentos básicos das grupoterapias. 2ed. Porto Alegre (RS): Artes Médicas; 2000.

7. Maximino VS. A constituição de grupos de atividades com pacientes graves. Rev Cent Est Ter Ocup 1995; 1(1): 27-32.

8. Munari DB, Rodrigues ARF. Enfermageme grupos. Goiânia (GO):A/B; 1997.

9. Ferrari AT. Metodologia da pesquisa científica. São Paulo (SP): Copyright; 1982.

10. Lasalle $P C$, Lasalle AJ. Grupos terapêuticos. In: Stuart GW, Laraia

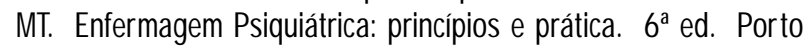
Alegre (RS): Artmed; 2001.

11. Venâncio J, organizador. Textos de apoio em saúde mental. 20a ed. Rio de Janeiro (RJ): FIOCRUZ; 2003.

12. Corrêa A K, Souza MCBM, Saeki T. Transição para o exercício profissional em enfermagem: uma experiência em grupo operativo. Esc Anna Nery Rev Enferm 2005; 9(3): 421 - 28.

13. Crippa CDGP. Grupo de espera para psicoterapia. Rev Spagesp 2002; (3):10-13.
Concordamos com as autoras no que diz respeito a necessidade de 0 enfermeiro buscar o preparo adequado para o bom desempenho de coordenação de grupos. Acreditamos que esse preparo começa com o auto-conhecimento e com conhecimentos específicos sobre dinâmica de grupo. Assim, possivelmente o recurso grupal poderá ser aplicado com efetiva segurança pelo enfermeiro. Neste sentido, ele estará valorizando a prática da Enfermagem na assistência aos pacientes e familiares.

14. Munari DB. Processo grupal em enfermagem: possibilidades e limites. Rev Bras Enferm 1997 jan/mar; 50(1): 37-52.

15. Colvero LA, Ide CAC. Rolim MA. Família e doença mental: a difícil convivência com a diferença. Rev Esc Enferm USP 2004 jun; 38(2): 197-205.

16.Birman J, Costa JF. Organização de instituições para uma psiquiatria comunitária. In: Amarante P, organizador. Psiquiatria social e psiquiatria reformada. Rio de Janeiro (RJ): Fiocruz; 1994.

17. Psicologia Grupal. Análisis Institucional. Escuela Pichon-Riviére. Grupos operativos. [online] 2004 [acesso 2004 out 11] Disponivel em: http://www.psicologiagrupal.cl

18. Furegato ARF. Grupos de auto- ajuda: psicóticos anônimos - para amigos e parentes dos psicóticos anônimos - A.P.P.A. Rev Spagesp 2002; (3): 126-129.

\section{Sobre as Autoras}

\section{Luciene Simöes Spadini}

Enfermeira Especialista em Enfermagem Psiquiátrica e Saúde Mental e Mestranda do Programa de Pós Graduação em Enfermagem Psiquiátrica da Escola de Enfermagem de Ribeirão Preto (EERP) I USP; e-mail: luspadini@ hotmail.com

\section{Maria Conceição Bernardo de Mello e Souza}

Professor Doutor do Departamento de Enfermagem Psiquiátrica e Ciências Humanas da EERP / USP; e-mail: consouza@ eerp.usp.br 